\title{
Altered microRNA dynamics in acute coronary syndrome
}

\author{
Ewelina Kazimierczyk ${ }^{1}$, Andrzej Eljaszewicz ${ }^{2}$, Remigiusz Kazimierczyk ${ }^{1}$, Marlena Tynecka², Paula Zembko², \\ Ewa Tarasiuk ${ }^{1}$, Karol Kaminski ${ }^{1,3}$, Bozena Sobkowicz ${ }^{1}$, Marcin Moniuszko, ${ }^{2,4}$, Agnieszka Tycinska ${ }^{1}$
}

${ }^{1}$ Department of Cardiology, Medical University of Bialystok, Bialystok, Poland

2Department of Regenerative Medicine and Immune Regulation, Medical University of Bialystok, Bialystok, Poland

${ }^{3}$ Department of Population Medicine and Civilization Diseases Prevention, Medical University of Bialystok, Bialystok, Poland

${ }^{4}$ Department of Allergology and Internal Medicine, Medical University of Bialystok, Bialystok, Poland

Adv Interv Cardiol 2020; 16, 3 (61): 287-293

DOI: https://doi.org/10.5114/aic.2020.99263

\begin{abstract}
A bstract
Introduction: In the course of acute myocardial infarction (AMI) cardiomyocyte injury, activation and destruction of endothelial cells together with inflammation lead to miRNA expression alterations.

Aim: To assess levels of circulating cardiac-specific (miR-1) and endothelial-specific (miR-126) miRNAs in the acute phase of AMI and after a follow-up period.

Material and methods: Seventeen AMI patients (mean age: $64.24 \pm 13.83$ years, mean left ventricle ejection fraction (LVEF): 42.6 $\pm 9.65 \%$ ), treated with primary percutaneous coronary intervention within the first $12 \mathrm{~h}$, had plasma miRNAs isolated (quantitative real-time PCR, Exiqon) on admission and after $19.2 \pm 5.9$ weeks. Measurements were also performed in a control group of healthy volunteers matched for age and sex.

Results: Concentrations of both miRNAs were significantly higher in AMI patients as compared to healthy controls: miR-1: 5.93 (3.15-14.92) vs. 1.46 (0.06-2.96), $p=0.04$; miR-126: 4.5 (3.11-7.64) vs. $0.54(0.36-0.99), p=0.00003$, respectively. Levels of both miRNAs significantly decreased after the follow-up period: miR-1: 5.93 (3.15-14.92) vs. 1.34 (0.04-2.34), $p=0.002$; miR-126: 4.5 $(3.11-7.64)$ vs. $1.18(0.49-1.68), p=0.0005)$. Moreover, miR-1 correlated positively with maximal troponin I concentration $(r=0.59$, $p=0.02)$ and negatively with $\operatorname{LVEF}(r=-0.76, p=0.0004)$.

Conclusions: In our study, miR-1 emerged as a marker of cardiomyocyte injury and loss of myocardial contractility, whereas dynamics of miR-126 concentration may reflect endothelial activation and damage in the most extreme stage of atherosclerosis, followed by angiogenesis in ischemic myocardium. However, to fully elucidate the role of miR-1 and miR-126 as biomarkers of AMI and future therapeutic targets, further research is required.
\end{abstract}

Key words: microRNA, myocardial infarction, miR-126, miR-1.

Su m m a ry

In the course of acute myocardial infarction (AMI) cardiomyocyte injury, endothelial damage and activation, as well as inflammation lead to miRNA expression alterations. However, reproducible research results on the subject are still lacking. This study revealed elevated levels of circulating cardiac-specific (miR-1) and endothelial-specific (miR-126) miRNAs in the acute phase of AMI compared to healthy volunteers, which decreased after a follow-up period of 19.2 weeks. Therefore, altered miR-1 and miR-126 levels may probably serve as biomarkers of AMI or future therapeutic targets.

\section{Introduction}

miRNAs are small, endogenous, noncoding RNAs which regulate the expression of different genes at the posttranscriptional level through binding to the particular complementary region of target messenger RNA (mRNA) and initiating its degradation [1]. It was previously con- firmed that miRNAs may be actively and selectively secreted by different cells and can be detected in various body fluids [2]. Circulating miRNAs are transported to recipient cells in complexes with proteins and high-density lipoproteins or encapsulated into microvesicles, which protects them from RNase activity [3]. Changes in circulating miRNA profiles and levels were shown recently,

\section{Corresponding author:}

Ewelina Kazimierczyk MD, PhD, Department of Cardiology, Medical University of Bialystok, Bialystok, Poland, e-mail: e-kazimierczyk@wp.pl Received: 23.03.2020, accepted: 9.05.2020. 
by our and other groups, to correlate with a number of pathological conditions, including cardiovascular disease and acute myocardial infarction. Therefore, it is believed that they may serve as markers of disease progression, treatment response and even the healing process of myocardial infarction, among other $[4,5]$.

To date, the most reliable and commonly used markers of ischemia are troponins [6]. Unfortunately, they are detectable in elevated concentrations only after 3-6 h from the onset of acute myocardial infarction (AMI) symptoms, which hinders early diagnosis and requires repetitive measurements with prolonged patient observation on emergency department [7]. Although the discovery of high-sensitivity cardiac troponins significantly improved AMI diagnosis, their elevated concentration still cannot exclusively indicate cardiac ischemic injury and may be associated with other cardiac and non-cardiac pathologies.

Nowadays, evaluation of molecular aspects of $\mathrm{AMI}$ has led to the assessment of different miRNAs, both in circulation and in cardiac tissue. Notably, changes in circulating miRNA profiles are caused not only by their passive leakage from the injured myocardium but also their functional release from circulating and locally activated leukocytes, platelets and endothelial cells, among others [2].

miR-1 is recognized as one of the cardiac-specific mRNAs, which is mainly expressed in cardiomyocytes and to a lesser extent in skeletal muscle cells [3]. miR-1 function is associated with cardiogenesis [8], prevention of cardiac hypertrophy [9] as well as the regulation of cardiac conduction system activity [10] and cardiomyocytes' apoptosis [11, 12]. miR-1 overexpression, however, turned out to have a detrimental effect on the heart - leading to destruction of sarcomere assembly through downregulation of genes encoding cardiac calmodulin protein (CALM 1 and 2) and cardiac myosin light chain kinase (MYLK3) [13]. miR-1 supports apoptosis through targeting anti-apoptotic genes $[11,12]$ as well as slow cardiac conduction through repressing KCNJ2 (which encodes the K+ channel subunit Kir2.1) and GJA1 (which encodes connexin 43), which results in arrhythmogenesis [10].

There is a growing body of evidence that in the course of AMI, circulating miRNAs do not only originate from the injured myocardium but also are regulated by inflammatory signals and are capable of further modification of inflammatory responses [3].

miR-126, referred to as endothelial-specific miRNA, was considered as a biomarker of endothelial activation and damage [14]. miR-126 was shown to maintain vascular integrity as well as endothelial homeostasis by downregulating angiogenesis inhibitors (SPRED-1 sprout-related protein 1) [15]. Furthermore, its release in microparticles from apoptotic and activated endothelial cells mediates production of $\mathrm{C}-\mathrm{X}-\mathrm{C}$ motif chemokine ligand 12 (CXCL12), which leads to progenitor cell recruitment and consequently endothelial repair [16]. Moreover,
miR-126 inhibits the expression of vascular cell adhesion molecule-1 (VCAM1) in the endothelium and attenuates production of monocyte chemoattractant protein-1 (MCP-1/CCL2) in the human monocyte/macrophage cell line and therefore may inhibit monocyte/macrophage infiltration [17]. More importantly, miR-126 is induced by proinflammatory signaling in immune cells and acts as a suppressor of proinflammatory responses by regulation of tumor necrosis factor (TNF) and angiotensin II production, among others [17].

\section{Aim}

There is still a substantial need to search for novel biomarkers allowing early AMI diagnosis and the assessment of the risk of heart failure progression. Observed changes among circulating miRNAs in AMI and determination of their function may result in new therapeutic approaches. Recently, we observed a significant increase of leukocyte-specific miRNA (miR-155 and miR-146) concentrations in the acute phase of $A M I$, which decreased after the follow-up period [4].

In this study, we aimed to determine plasma concentrations of cardiac-specific miR-1 and endothelial-specific miR-126 in AMI patients, as both miRNAs probably reflect different aspects of cardiovascular injury.

\section{Material and methods}

\section{Patient characteristics}

Seventeen patients ( $64.6 \pm 13.8$ years old, 4 females) were enrolled in the study (Table I). The study group was recruited from patients admitted to the Department of Cardiology with diagnosis of AMI. The criteria for AMI were as follows [18]: detection of rise and/or fall of cardiac biomarker values (troponin) with at least one value above the $99^{\text {th }}$ percentile of the upper reference limit and with at least one of the following: 1) symptoms of ischemia; 2) new or presumably new significant ST-T changes or new left bundle branch block (LBBB); 3) development of pathological Q waves in the ECG; 4) imaging evidence of new loss of viable myocardium, or new regional wall motion abnormality. Exclusion criteria were as follows: 1) a history of severe pre-existing cardiac disease; 2) $\mathrm{AMI}$ for $>24 \mathrm{~h}$ before onset; 3) unstable angina pectoris/myocardial infarction in a period of 6 months; 4) chronic auto-inflammatory disease; 5) active inflammatory process in a period of a month; 6) a history of proliferative disease; 7 ) serious renal disease requiring dialysis; 8) age below 18 years.

The assessed clinical parameters included age, sex, and coronary risk factors (smoking, hypertension, diabetes mellitus, hyperlipidemia, obesity). All subjects received typical pharmacotherapy of myocardial infarction (according to ESC guidelines) and underwent coronary angiography during the first day of hospitalization. Significant coronary stenosis was defined as $\geq 75 \%$. 
The control group consisted of nine healthy volunteers adjusted for age and sex.

The study was approved by the institutional medical ethics committee. All subjects gave informed consent for participating in the study, including taking and storage of blood samples. The study complied with the Declaration of Helsinki.

\section{Blood collection and specimen preparation}

$15 \mathrm{ml}$ of venous blood was collected into EDTA containing vials on admission (before angiography and coronary stenting) and processed immediately, namely centrifuged for $15 \mathrm{~min}$ at $1000 \times \mathrm{g}$ within $30 \mathrm{~min}$ of collection and were stored at $-80^{\circ} \mathrm{C}$ until analysis.

Furthermore, all the patients had routine laboratory tests, measured using standard methods: morphology, troponin I, creatinine kinase, C-reactive protein, parameters of renal function (creatinine concentration, glomerular filtration rate), lipids and brain natriuretic peptide concentrations (BNP).

After follow-up period of $19.2 \pm 5.9$ weeks blood samples were collected again and processed according to the protocol.

\section{RNA isolation and quantitative polymerase chain reaction analysis}

RNA from previously collected plasma samples was isolated using miRCURY RNA Isolation Kit Biofluids (Exiqon) according to the manufacturer's instructions. The RNA spike-in controls (Exiqon) were used according to the manufacturer's instruction for validation of RNA isolation (UNiSp2, UNiSp4 and UNiSp5), cDNA synthesis and PCR amplification (UniSp6, cel-miR-39-3p). Moreover, an additional template-free control was purified by the samples and profiled. Briefly, EDTA-plasma samples were thawed on melting ice and centrifuged to avoid presence of cell debris. Next, debris-free samples were lysed using lysing solution and RNA isolation was performed. Isolated RNA was reverse-transcribed using a miRCURY LNA Universal RT cDNA Synthesis kit (Exiqon). The cDNA was diluted 50× and assayed according to the protocol for the miRCURY LNA Universal RT microRNA PCR system (Exiqon). All LNA primers were obtained from Exiqon. The Step-One Plus system was used for amplification (Applied Biosystems). All data were normalized to the cel-miR-39-3p control.

Results were derived by the $\left(2^{-\triangle C T}\right) \times 10^{4}(\mathrm{miR}-1)$ or $\left(2^{-\Delta C T}\right) \times 10^{2}($ miR-126) and normalized to the volume and to the expression of cel-miR-39-p3.

\section{Statistical analysis}

Data are presented as median with inter-quartile ranges (IQR) for non-normally distributed variables or mean \pm standard deviation (SD) for normally distributed continuous variables. Categorical variables were displayed as numbers and percentages. The statistical
Table I. Patients' baseline characteristics

\begin{tabular}{|c|c|}
\hline Parameter & Results \\
\hline \multicolumn{2}{|l|}{ Demographic and clinical: } \\
\hline Age [years] & $64.24 \pm 13.83$ \\
\hline Female, \% (n) & $23.5(4)$ \\
\hline Weight [kg] & $77.12 \pm 12.9$ \\
\hline $\mathrm{BMI}\left[\mathrm{kg} / \mathrm{m}^{2}\right]$ & $25.9 \pm 3.5$ \\
\hline \multicolumn{2}{|l|}{ NYHA class, \% (n): } \\
\hline 1 & $47.06(8)$ \\
\hline$\|$ & $47.06(8)$ \\
\hline III & $5.88(1)$ \\
\hline Systolic blood pressure [mm Hg] & $152.82 \pm 14.99$ \\
\hline Heart rate [beats/min] & $79.06 \pm 13.83$ \\
\hline $\begin{array}{l}\text { Previously diagnosed CAD (ACS/PCI > } 6 \text { months } \\
\text { ago), \% (n) }\end{array}$ & $17.65(3)$ \\
\hline Previously diagnosed heart failure, \% (n) & $11.76(2)$ \\
\hline Atrial fibrillation, \% ( $n$ ) & $5.88(1)$ \\
\hline Arterial hypertension, \% ( $n)$ & $35.29(6)$ \\
\hline Hyperlipidemia, \% ( $n$ ) & $29.41(5)$ \\
\hline Diabetes, \% (n) & $5.88(1)$ \\
\hline \multicolumn{2}{|l|}{ Laboratory results: } \\
\hline CRP $[I U / I]$ & $7.1(6-37)$ \\
\hline Troponin $\max [\mathrm{ng} / \mathrm{l}]$ & $9.7(4.7-41.9)$ \\
\hline Creatinine $[\mathrm{mg} / \mathrm{dl}]$ & $0.97 \pm 0.26$ \\
\hline Total cholesterol [mg/dl] & $192.94 \pm 26.07$ \\
\hline $\mathrm{LDL}[\mathrm{mg} / \mathrm{dl}]$ & $118 \pm 30.1$ \\
\hline Hemoglobin [g\%] & $13.9 \pm 1.19$ \\
\hline Fasting glucose [mg/dl] & $108.47 \pm 17.26$ \\
\hline Glucose on admission [mg/dl] & $141.77 \pm 34.46$ \\
\hline \multicolumn{2}{|l|}{ Echocardiography: } \\
\hline LVEF, \% & $42.6 \pm 9.65$ \\
\hline WMSI & $1.77 \pm 0.55$ \\
\hline LVIDd [cm] & $4.91 \pm 0.42$ \\
\hline$\overline{L A}[\mathrm{~cm}]$ & $4 \pm 0.4$ \\
\hline Left ventricular mass index & $114.25 \pm 18.04$ \\
\hline E/A ratio & $1.06 \pm 0.34$ \\
\hline
\end{tabular}

Data are presented as mean \pm standard deviation or median and interquartile range (IQR). ACS - acute coronary syndrome, BMI - body mass index, CAD coronary artery disease, $C R P$ - C-reactive protein, $E / A$ - ratio of early $(E)$ to late (A) ventricular filling velocities, $L A$ - left atrium, $L D L$ - low-density cholesterol, LVEF - left ventricular ejection fraction, LVIDd - left ventricular end-diastolic dimension, NYHA - New York Heart Association, PCI - percutaneous coronary intervention, WMSI - wall motion score index. 
analysis was performed using Student's t-test or the Mann-Whitney $U$ test for continuous data and the $\chi^{2}$ test for categorical variables. The Wilcoxon signed-rank test was used to compare repeated measurements. Pearson's or Spearman's correlation coefficient was used to examine the relationship between two continuous variables. $P<0.05$ was deemed statistically significant. The statistical software package Statistica 10 (StatSoft Polska) was used for the analysis.

\section{Results}

Most of the enrolled subjects were patients with the diagnosis of ST-segment myocardial infarction (STEMI) $(88 \%, n=15)$. Blood samples were collected at a median time of $4 \mathrm{~h}$ (IQR: 2-9) after the onset of symptoms.

On admission, we observed elevated markers of myocardial necrosis (mean troponin I concentration was 9.7 IU/I (4.7-41.9), normal value: $0-0.3 \mathrm{ng} / \mathrm{l})$ as well as of inflammation (C-reactive protein (CRP) - $41.71 \pm 99.1 \mathrm{U} / \mathrm{l}$, white blood cell count $-11.46 \pm 3.85 \times 10^{3} / \mu \mathrm{l}$ ). All patients had preserved renal function with the mean glomerular filtration rate $85.94 \pm 27.59 \mathrm{ml} / \mathrm{min}$. Low-density lipoprotein $(\mathrm{LDL})$ remained in a normal range, although the level was too high concerning patients with increased cardiovascular risk (118 $\pm 30.1 \mathrm{mg} / \mathrm{dl})$. Mean glucose concentration on admission was $141.77 \pm 34.46 \mathrm{mg} / \mathrm{dl}$ and none of the patients had obesity (body mass index (BMI) was $25.9 \pm 3.5 \mathrm{~kg} / \mathrm{m}^{2}$ ).

All the patients had echocardiography performed during the first $24 \mathrm{~h}$. Mean left ventricle ejection fraction (LVEF) was $42.6 \pm 9.65 \%$ (Table I).

Both miRNA levels were significantly higher in AMI patients on admission as compared to healthy controls: miR-1: $5.93(3.15-14.92)$ vs. 1.46 (0.06-2.96), $p=0.04$; miR-126: 4.5 (3.11-7.64) vs. 0.54 (0.36-0.99), $p=0.00003$, respectively.

Furthermore, levels of both analyzed miRNAs significantly decreased after the follow-up period: miR-1: 5.93 (3.15-14.92) vs. $1.34(0.04-2.34), p=0.002$ and miR-

A

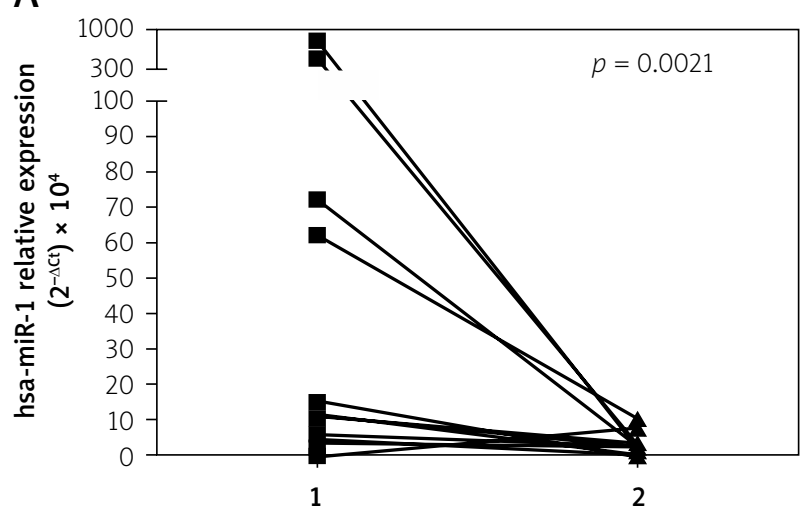

126: 4.5 (3.11-7.64) vs. $1.18(0.49-1.68), p=0.0005)$ (Figure 1) and achieved values comparable to the one observed in healthy controls (miR-1: 1.34 (0.04-2.34) vs. 1.46 (0.06-2.96), $p=0.87$ and miR-126: 1.18 (0.49-1.68) vs. 0.54 (0.36-0.99), $p=0.38)$.

miR-1 correlated with maximal troponin I (cTnl) concentration $(r=0.59, p=0.02)$ and negatively with LVEF $(r=-0.76, p=0.0004)$ (Figure 2$)$. We did not reveal any significant correlation between miR-126 level and maximal cTnl $(r=0.25 ; p=0.35)$. miR-1 and miR-126 levels did not significantly correlate with age, BMI, systolic blood pressure or laboratory results including CRP, creatinine, lipids or glucose concentrations. Furthermore, we did not reveal any correlations with other comorbidities.

\section{Discussion}

In this study, we observed that both miR-1 and miR126 levels were significantly higher in AMI patients at admission as compared to the healthy controls. Furthermore, in the acute phase of AMI, miR-1 correlated positively with cTnl concentration and negatively with LVEF. In the following months, after the follow-up period, levels of both analyzed miRNAs significantly decreased to the values observed in the control group.

miRNAs may become important markers in diagnosis and prognosis of AMI due to their stability in circulation [19]. However, it requires further research and overcoming remaining limitations, such as duration of the assay, lack of standardized normalization methods, unknown reference ranges and influence of concomitant medication and comorbidities [20]. Interestingly, we did not find any correlation with other clinical confounders.

miR-126 and miR-1 are highly expressed in the cardiovascular system and their plasma concentrations are therefore affected by cardiovascular pathology. We observed elevated levels of circulating cardiac-delivered miR-1 as well as vascular-delivered miR-126 in AMI patients at baseline compared to controls.

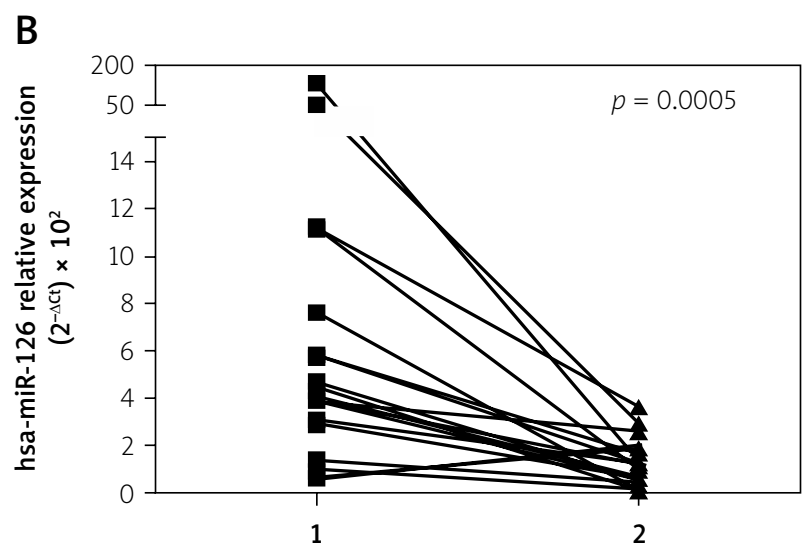

Figure 1. Changes in miR-1 (A) and miR-126 (B) concentration in patients admitted to hospital with diagnosis of AMI (1) and in follow-up period (2). To compare repeated measurements the Wilcoxon signed-rank test was used 
A

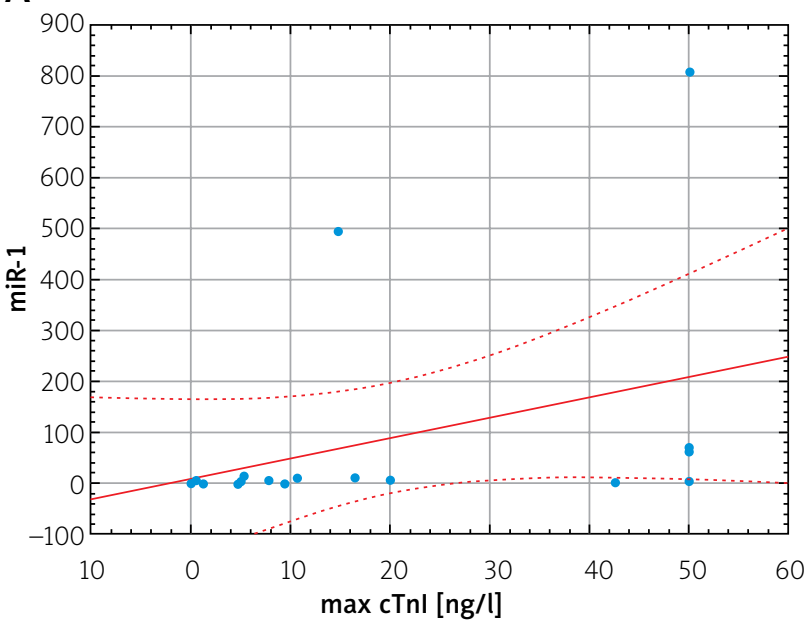

B

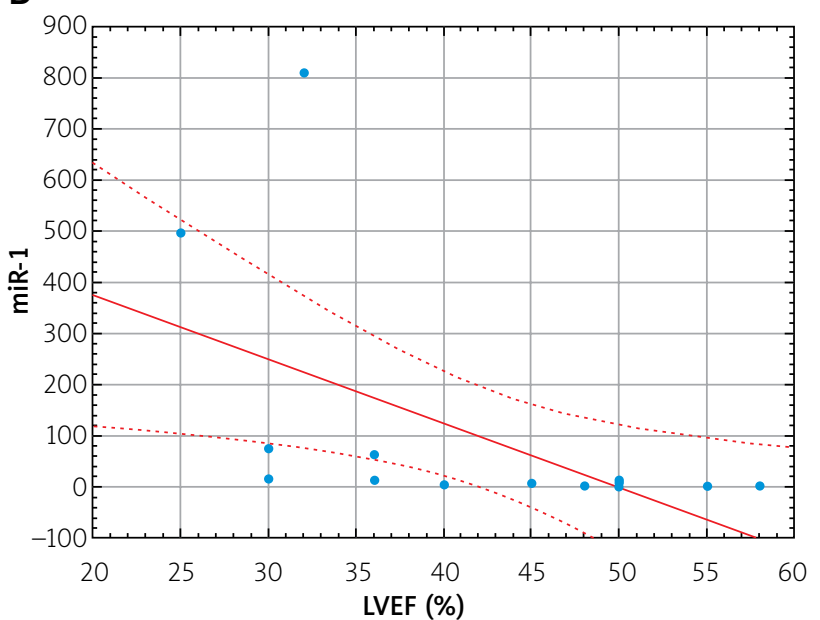

Figure 2. Correlation of miR-1 concentration with max cTnl (A) and LVEF (B)

cTnl - cardiac troponin I, LVEF - left ventricle ejection fraction, miR - microRNA.

Previously, miR-1 level was also shown to increase in blood after STEMI, NSTEMI or unstable angina [21, 22], probably due to the passive release from necrotic cardiomyocytes. Elevated miR-1 blood levels were measured within $4 \mathrm{~h}$ after the onset of symptoms [21], reaching even a 100-300-fold increase within $12 \mathrm{~h}$ and reaching baseline values after 3 days [23]. However, peak concentration of mir-1 was documented even $2.5 \mathrm{~h}$ after the onset of AMI [24].

Furthermore, the positive correlation of miR-1 with the concentration of maximal cTnl may also indicate a similar mechanism of their release [25]. Previous findings analyzing cardiac ischemic biomarkers, apart from correlation with cardiac troponins, also revealed positive correlation with creatine kinase MB (CK-MB) [23]. However, evaluation of the diagnostic value of miR-1 in comparison to well-established troponins (assessed both with standard and high-sensitive assays) ended with various results. Cardiac-specific miRNAs, including miR-1, miR-133, miR-208, or miR-499, showed no added diagnostic value to troponins [22, 25], comparable sensitivity [26], or significantly higher diagnostic value than troponins [27]. Among the investigated miRNAs the highest accuracy in AMI diagnosis was attributed to miR-499 and miR-208, which are selectively expressed in cardiomyocytes, released within an hour from the onset of symptoms, and are immeasurable in healthy humans [28]. Eventually, combining all cardiac-specific miRNAs with high-sensitivity troponin or miRNA ratio application resulted in significantly improved diagnostic value [22]. Therefore, as previous studies described, early detection of elevated levels of circulating miR-1 in AMI, which preceded the peak of cardiac troponin release [24, 28], may be complementary to troponin measurement. Thus, this may allow for an earlier diagnosis and differentiation from other troponin-positive conditions.
Furthermore, the observed negative correlation between miR-1 and LVEF indicates an association of increased levels of circulating plasma miR-1 with the impairment of cardiac function. Probably, miR-1 overexpression results in damage of sarcomere assembly [29], impairs cardiac conduction system activity [10], enhances cardiomyocytes apoptosis [11] and decreases resistance to reactive oxygen species (ROS) [12]. Indeed, it was documented that oxidative stress increases miR-1 concentration and as a result decreases the expression of heat shock proteins [12]. This hypothesis, however, needs to be further elucidated. Furthermore, Zhang et al. revealed the negative correlation of miR-1 levels with LVEF, ratio of the early (E) to late $(A)$ ventricular filling velocities (E/A ratio), as well as left ventricular fractional shortening (FS\%), and concluded that the adverse effect of mir- 1 is associated with impaired systolic as well as diastolic function [29].

Several previous reports have documented a substantial decrease of circulating miR-126 in stable coronary artery disease (CAD) [30,31] or AMI patients [16], compared to healthy controls. Furthermore, among CAD patients, lower miR-126 levels were observed in the case of multi-vessel disease and in patients with a higher major adverse cardiovascular event rate [30, 32]. These results support the hypothesis that miR-126 maintains a proliferative reserve in endothelial cells preventing atherosclerotic lesion formation, whereas the observed loss of miR-126 reflects abnormal endothelial and platelet function in CAD patients [31].

Contradicting these findings, D'Alessandra et al. reported elevated miR-126 plasma levels in both CAD and unstable angina and documented their good diagnostic value [33]. Increased levels of miR-126 were also observed in patients with acute coronary syndrome compared to patients with stable CAD [34] or after limb ischemia in- 
duced with thigh cuff inflation in healthy volunteers [35] Similarly, here we found significantly elevated levels of miR-126 in AMI patients compared to healthy controls. However, after the follow-up miR-126 levels decreased and reached normal levels.

Notably, AMI results in endothelial damage as well as systemic endothelial activation. Thus, elevated levels of circulating miR-126 may result from vascular injury and active secretion of miR-126-enriched microparticles [16, 36]. miR-126 inhibits endothelial cells apoptosis and, through up-regulation of endothelial CXCL12, contributes to circulating progenitor cells mobilization and endothelial repair [16]. Moreover, previous reports indicated that hypoxia stimulates miR-126 expression not only in endothelial cells but also in other cell types, such as vascular smooth muscle cells or cardiomyocytes [37, 38]. In cardiac muscle cells, miR-126 was found to stimulate myogenesis and proliferation as well as inducing proangiogenic and pro-survival signaling. As miR-126 contributes to the process of repair through enhancing angiogenesis and progenitor cell recruitment, it may be taken up by the vasculature at the sites of injury. Notably, levels of circulating miR-126 decreased during transcoronary passage through the culprit vessel, suggesting its consumption [36].

Apart from endothelium, miR-126 has a much lower concentration in platelets and is released to the circulation as a consequence of their activation and thrombosis $[35,39]$. However, platelet-derived miR-126 was found to be decreased after aspirin administration [39]. This may partially explain the decrease of miR-126 levels in the months after AMI or in stable CAD patients [31] as a result of received antiplatelet therapy. Furthermore, inflammation restriction in the months after $A M I$ results in decreased levels of markers associated with endothelial injury and hypoxia.

The results presented above may have some clinical implications for the future. Although unequivocal results on the predominance of miRNAs over troponins are lacking, the assessment of miRNA levels may be complementary to troponins for AMI diagnosis. Unfortunately, the main obstacle for miRNAs routine application is the technique of detection. Although there is a growing number of newly developed methods of miRNA assessments, most studies that focused only on one or two miRNAs opted for traditional qPCR as the gold standard assay. Despite high sensitivity and specificity, this analysis technique is complex, time-consuming and expensive. Moreover, it still needs the determination of a unified normalization method and each of miRNA standard range. Hopefully, with the rapid development of new techniques, we will be able to routinely assess miRNA levels in AMI patients.

Apart from its diagnostic value, the evaluation of miRNAs may provide novel methods of treatment. The application of miRNA mimics may result in upregulation of miR-126 endothelial expression, leading to improved myocardial regeneration. On the other hand, upregulation of miR-1 may prevent cardiomyocyte hypertrophy. The inhibition of miR-1 overexpression with antisense oligonucleotides probably may prevent cardiomyocytes apoptosis, myofibrils destruction or arrythmias. However, their introduction to routine practice requires further research.

The main limitation of the study is the relatively small size of the study group, which results from strict inclusion criteria and numerous exclusion criteria. However, patients who finally met the criteria were comprehensively assessed for their molecular profile using the latest available techniques and the results were compared to a control group. Additionally, we did not assess the levels of miRNAs in myocardial tissue or in the coronary arteries.

\section{Conclusions}

The alterations of circulating miRNAs reflect different aspects of cardiovascular pathology in the course of AMI. Myocardial ischemia probably induces a specific shift in circulating miRNAs, which are expressed by cardiomyocytes and related to myocardial cells or which are induced by other cells upon cardiac injury. Therefore, not only miRNAs associated with cardiac damage, but also linked to other conditions accompanying myocardial ischemia, may be useful as biomarkers of $\mathrm{AMI}$ or future therapeutic targets.

\section{Acknowledgments}

Grant provided by Medical University of Bialystok, Poland, No. 133-53833L and 143-53645L.

\section{Conflict of interest}

The authors declare no conflict of interest.

\section{References}

1. Lagos-Quintana M, Rauhut R, Lendeckel W, et al. Identification of novel genes coding for small expressed RNAs. Science 2001; 294: 853-8.

2. Turchinovich A, Weiz L, Langheinz A, et al. Characterization of extracellular circulating microRNA. Nucleic Acids Res 2011; 39: 7223-33.

3. Deddens JC, Colijn JM, Oerlemans MI, et al. Circulating microRNAs as novel biomarkers for the early diagnosis of acute coronary syndrome. J Cardiovasc Transl Res 2013; 6: 884-98.

4. Kazimierczyk E, Eljaszewicz A, Zembko P, et al. The relationships among monocyte subsets, miRNAs and inflammatory cytokines in patients with acute myocardial infarction. Pharmacol Rep 2019; 71: 73-81.

5. Wang R, Li N, Zhang Y, et al. Circulating microRNAs are promising novel biomarkers of acute myocardial infarction. Intern Med 2011; 50: 1789-95.

6. Mair J, Artner-Dworzak E, Lechleitner P, et al. Cardiac troponin T in diagnosis of acute myocardial infarction. Clin Chem 1991; 37 : 845-52.

7. Keller T, Zeller T, Peetz D, et al. Sensitive troponin I assay in early diagnosis of acute myocardial infarction. N Engl J Med 2009; 361: 868-77. 
8. Zhao Y, Samal E, Srivastava D. Serum response factor regulates a muscle-specific microRNA that targets Hand2 during cardiogenesis. Nature 2005; 436: 214-20.

9. Gladka MM, da Costa Martins PA, De Windt LJ. Small changes can make a big difference - microRNA regulation of cardiac hypertrophy. J Mol Cell Cardiol 2012; 52: 74-82.

10. Yang B, Lin H, Xiao J, et al. The muscle-specific microRNA miR-1 regulates cardiac arrhythmogenic potential by targeting GJA1 and KCNJ2. Nat Med 2007; 13: 486-91.

11. Tang $Y$, Zheng J, Sun $Y$, et al. MicroRNA-1 regulates cardiomyocyte apoptosis by targeting Bcl-2. Int Heart J 2009; 50: 377-87.

12. Xu C, Lu Y, Pan Z, et al. The muscle-specific microRNAs miR-1 and miR-133 produce opposing effects on apoptosis by targeting HSP60, HSP70 and caspase-9 in cardiomyocytes. J Cell Sci 2007; 120: 3045-52.

13. Ai J, Zhang R, Gao X, et al. Overexpression of microRNA-1 impairs cardiac contractile function by damaging sarcomere assembly. Cardiovasc Res 2012; 95: 385-93.

14. van Solingen C, Bijkerk R, de Boer HC, et al. The Role of microRNA-126 in vascular homeostasis. Curr Vasc Pharmacol 2015; 13: 341-51.

15. Wang S, Aurora AB, Johnson BA, et al. The endothelial-specific microRNA miR-126 governs vascular integrity and angiogenesis. Dev Cell 2008; 15: 261-71.

16. Zernecke A, Bidzhekov K, Noels $\mathrm{H}$, et al. Delivery of microRNA-126 by apoptotic bodies induces CXCL12-dependent vascular protection. Sci Signal 2009; 2: 81.

17. Harris TA, Yamakuchi $M$, Ferlito $M$, et al. MicroRNA-126 regulates endothelial expression of vascular cell adhesion molecule 1. Proc Natl Acad Sci USA 2008; 105: 1516-21.

18. Thygesen K, Alpert JS, Jaffe AS, et al.; the Writing Group on behalf of the Joint ESC/ACCF/AHA/WHF Task Force for the Universal Definition of Myocardial Infarction. Third universal definition of myocardial infarction. Eur Heart J 2012; 33: 2551-67.

19. Chen X, Ba Y, Ma L, et al. Characterization of microRNAs in serum: a novel class of biomarkers for diagnosis of cancer and other diseases. Cell Res 2008; 18: 997-1006.

20. Goretti E, Wagner DR, Devaux Y. miRNAs as biomarkers of myocardial infarction: a step forward towards personalized medicine? Trends Mol Med 2014; 20: 716-25.

21. Kuwabara, Y, Ono K, Horie T, et al. Increased microRNA-1 and microRNA-133a levels in serum of patients with cardiovascular disease indicate myocardial damage. Circ Cardiovasc Genet 2011; 4: 446-54.

22. Oerlemans MI, Mosterd A, Dekker MS, et al. Early assessment of acute coronary syn- dromes in the emergency department: the potential diagnostic value of circulating microRNAs. EMBO Mol Med 2012; 4: 1176-85.

23. Cheng Y, Tan N, Yang J, et al. A translational study of circulating cell-free microRNA-1 in acute myocardial infarction. Clin Sci 2010; 119: 87-95.

24. D’Alessandra Y, Devanna P, Limana F, et al. Circulating microRNAs are new and sensitive biomarkers of myocardial infarction. Eur Heart J 2010; 31: 2765-73.

25. Li YQ, Zhang MF, Wen HY, et al. Comparing the diagnostic values of circulating microRNAs and cardiac troponin $T$ in patients with acute myocardial infarction. Clinics 2013; 68: 75-80.

26. Liu G, Niu X, Meng X, et al. Sensitive miRNA markers for the detection and management of NSTEMI acute myocardial infarction patients. J Thorac Dis 2018; 10: 3206-15.
27. Lippi G, Mattiuzzi C, Cervellin G. Circulating microRNAs (miRs) for diagnosing acute myocardial infarction: meta-analysis of available studies. Int J Cardiol 2013; 167: 277-8.

28. Wang GK, Zhu JQ, Zhang JT, et al. Circulating microRNA: a novel potential biomarker for early diagnosis of acute myocardial infarction in humans. Eur Heart J 2010; 31: 659-66.

29. Zhang R, Niu H, Ban T, et al. Elevated plasma microRNA-1 predicts heart failure after acute myocardial infarction. Int J Cardiol 2013; 166: 259-60.

30. Jansen F, Yang X, Proebsting S, et al. MicroRNA expression in circulating microvesicles predicts cardiovascular events in patients with coronary artery disease. J Am Heart Assoc 2014; 3: e001249.

31. Fichtlscherer S, De Rosa S, Fox $\mathrm{H}$, et al. Circulating microRNAs in patients with coronary artery disease. Circ Res 2010; 107: 677-84.

32. Li HY, Zhao X, Liu YZ, et al. Plasma microRNA-126-5p is associated with the complexity and severity of coronary artery disease in patients with stable angina pectoris. Cell Physiol Biochem 2016; 39: 837-46.

33. D'Alessandra Y, Carena MC, Spazzafumo L, et al. Diagnostic potential of plasmatic microRNA signatures in stable and unstable angina. PLoS One 2013; 8: e80345.

34. De Rosa S, Eposito F, Carella C, et al. Transcoronary concentration gradients of circulating microRNAs in heart failure. Eur J Heart Fail 2018; 20: 1000-10.

35. Zampetaki A, Willeit P, Tilling L, et al. Prospective study on circulating microRNAs and risk of myocardial infarction. J Am Coll Cardiol 2012; 60: 290-9.

36. De Rosa S, Fichtlscherer S, Lehmann R, et al. Transcoronary concentration gradients of circulating microRNAs. Circulation 2011; 124: 1936-44.

37. Voellenkle C, Rooij JV, Guffanti A, et al. Deep-sequencing of endothelial cells exposed to hypoxia reveals the complexity of known and novel microRNAs. RNA 2012; 18: 472-84.

38. Shi $H$, Chen L, Wang $H$, et al. Synergistic induction of miR-126 by hypoxia and HDAC inhibitors in cardiac myocytes. Biochem Biophys Res Commun 2013; 430: 827-32.

39. de Boer HC, van Solingen C, Prins J, et al. Aspirin treatment hampers the use of plasma microRNA-126 as a biomarker for the progression of vascular disease. Eur Heart J 2013; 34: 3451-7. 